\title{
Dissection Aneurysm of the Radiculomedullary Branch of the Artery of Adamkiewicz With Subarachnoid Hemorrhage -Case Report-
}

\author{
Satoshi IIHOSHI, ${ }^{1}$ Kei MiYata, ${ }^{1}$ Tomohiro MURAKAmi, ${ }^{1}$ \\ Takahisa KANEKO, ${ }^{1}$ and Izumi KOYANAGI ${ }^{1}$
}

${ }^{1}$ Department of Neurosurgery, Sapporo Medical University School of Medicine, Sapporo, Hokkaido

\begin{abstract}
A 60-year-old female presented with sudden onset of severe headache and back pain, followed by nausea. The initial head computed tomography (CT) scan revealed posterior fossa subarachnoid hemorrhage (SAH). Spinal $\mathrm{T}_{2}$-weighted magnetic resonance imaging demonstrated $\mathrm{SAH}$, and a homogeneous and slightly low signal intensity mass at T11. Spinal angiography in the early arterial phase revealed a small pearl and string-like aneurysm of the proximal radiculomedullary artery on the left side at the T12 level. Forty days after the onset of SAH, CT angiography demonstrated complete occlusion of the dissecting aneurysm and the preserved anterior spinal artery. The present case of ruptured dissecting aneurysm of the radiculomedullary branch of the artery of Adamkiewicz with SAH underwent subsequent spontaneous occlusion, indicating that the wait-and-see strategy may be justified and will provide adequate treatment.
\end{abstract}

Key words: dissection aneurysm, Adamkiewicz artery, anterior spinal artery, subarachnoid hemorrhage, treatment

\section{Introduction}

Spinal subarachnoid hemorrhage (SAH) is a very rare event and accounts for less than $1 \%$ of all reported cases of SAH.) Spinal SAH is usually associated with medullar tumors, such as neurinomas, ependymomas, hemangioblastomas, ${ }^{2,6)}$ or spinal arteriovenous malformation (AVM), which can lead to formation of an aneurysm in a feeding artery. Isolated spinal artery aneurysms (not associated with AVM or tumor) are extremely rare. We report a case of a ruptured dissection aneurysm of the radiculomedullary branch of the artery of Adamkiewicz associated with SAH.

\section{Case Report}

A 60-year-old female patient suddenly developed severe headache, back pain, and left lower limb pain, followed by nausea. On admission, she was awake, orientated, and cooperative, had moderate neck stiffness, and had a Glasgow Coma Scale score of 15. Her initial blood pressure was $170 / 90 \mathrm{mmHg}$. She had no other neurological deficits. She had a history of hypertension and hyperlipidemia, but no predisposing clinical factors were identified. Laboratory examination showed no abnormal findings suggestive

Received December 14, 2010;

Accepted February 28, 2011 of inflammation or infection. Autoimmune diseases were excluded because she had no relevant history or specific clinical symptoms.

Initial head computed tomography (CT) revealed SAH, with blood in the cerebellomedullary, pontocerebellar, and prepontine cisterns, as well as in the fourth ventricle. CT angiography of the head showed no definitive evidence of aneurysm or vascular malformation. To investigate a possible spinal AVM or mass lesion, magnetic resonance (MR) imaging of the spine was performed with and without contrast medium, and demonstrated extensive $\mathrm{SAH}$ of the cervical and thoracic spinal cord. $\mathrm{T}_{2}$ weighted MR imaging revealed a homogeneous and slightly low signal intensity mass lesion at the T11 vertebral body level (Fig. 1). Digital subtraction angiography of the spinal vessels on the next day showed no definitive evidence of aneurysm or vascular malformation (Fig. 2). Follow-up digital subtraction angiography and CT angiography 20 days after SAH demonstrated a small pearl and string-like aneurysm of a radiculomedullary branch of the left intercostal artery at the T12 level with a diameter of approximately $2 \times 8 \mathrm{~mm}$ (Figs. 3 and 4 ). However, the radiculomedullary branch of the artery of Adamkiewicz, and arteriovenous shunts or abnormal veins were not seen. The interval change in the signal characteristics raised concern for an aneurysm of the anterior spinal artery with partial thrombosis. 

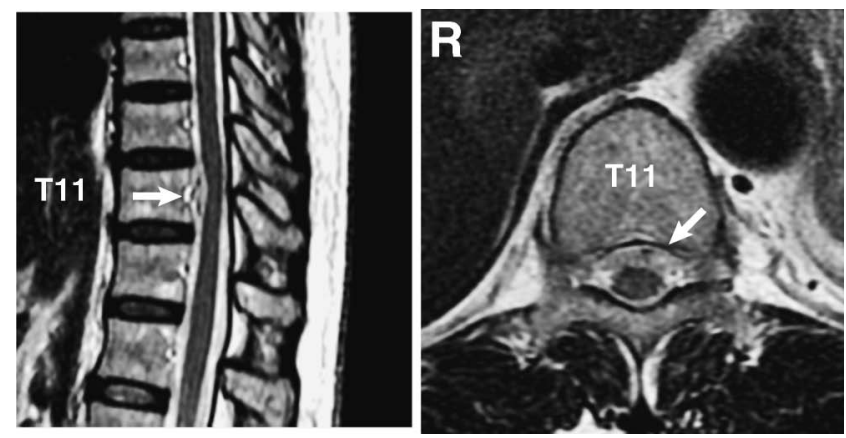

Fig. 1 Sagittal (left) and axial (right) $\mathrm{T}_{2}$-weighted magnetic resonance images revealing a homogeneous and slightly low signal intensity mass (arrow) at the T11 level.

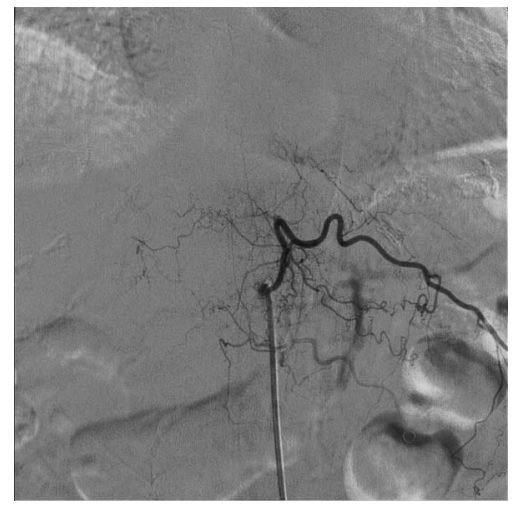

Fig. 2 Control digital subtraction angiogram of the segmental artery at the left T12 level showing no definitive evidence of aneurysm or vascular malformation.
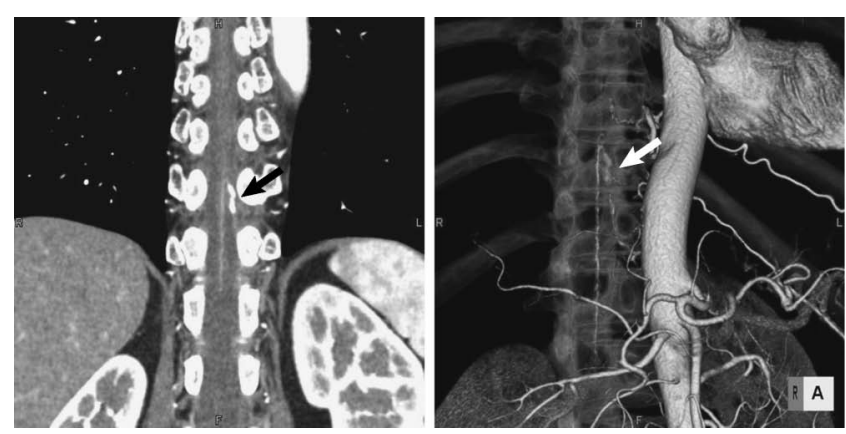

Fig. 3 Coronal (left) and three-dimensional reconstruction (right) computed tomography angiograms 20 days after subarachnoid hemorrhage demonstrating a small pearl and string-like aneurysm (arrow) of a radiculomedullary branch of the artery of Adamkiewicz originating from the left side of T12 level with a diameter of approximately $2 \times 8 \mathrm{~mm}$.

The findings and treatment options were discussed with the patient's family, and the neurosurgical and endovascular teams. We refrained from performing operative clipping or endovascular coiling and trapping of the
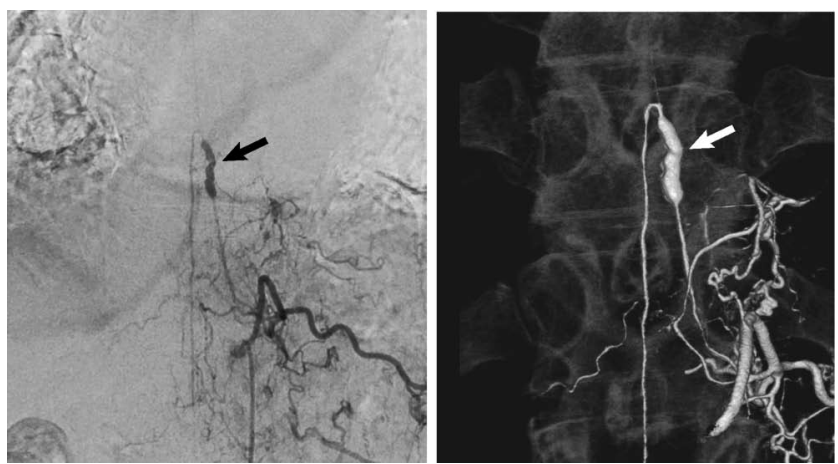

Fig. 4 Left: Posteroanterior digital subtraction angiogram 20 days after subarachnoid hemorrhage showing a fusiform aneurysm (arrow) of the radiculomedullary branch of the artery of Adamkiewicz originating from the left side of T12 level. The aneurysm occurs immediately before the radiculomedullary artery reaches the longitudinal anterior axis supplying the anterior spinal artery. Right: Three-dimensional rotational spinal angiogram demonstrating the aneurysm (arrow) of the artery of Adamkiewicz originating from the left side of T12 level.

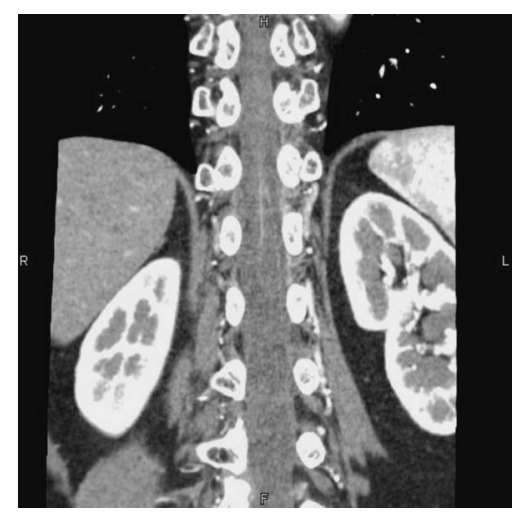

Fig. 5 Coronal computed tomography angiogram 40 days after subarachnoid hemorrhage demonstrating complete occlusion of the dissecting aneurysm and the preserved anterior spinal artery.

aneurysm because of the high risk of occluding the anterior spinal artery during the intervention. Forty days after the onset of SAH, CT angiography demonstrated complete occlusion of the dissecting aneurysm and the preserved anterior spinal artery (Fig. 5). During hospitalization, the patient recovered completely and was discharged without new neurological deficits. About 3 months after the initial onset of clinical symptoms, CT angiography of the spinal vessels was again performed to assess the effect of conservative treatment. The previous dissecting aneurysm could no longer be detected. The patient's subsequent clinical follow up was uneventful.

\section{Discussion}

AVM, arteriovenous fistula (AVF), aneurysm, and spinal cord tumor can cause spinal SAH. Spinal SAH has been 
Table 1 Reported cases of ruptured isolated spinal artery aneurysms of the radiculomedullary branch of the artery of Adamkiewicz

\begin{tabular}{|c|c|c|c|c|c|}
\hline Author (Year) & Age (yrs)/Sex & Location & Clinical symptoms & Management & Glasgow Outcome Scale \\
\hline Garcia et al. $(1979)^{5)}$ & $34 / \mathrm{F}$ & T6 & paraplegia, headache & not available & 1 (death) \\
\hline Vishteh et al. $(1997)^{20)}$ & $30 / \mathrm{M}$ & T11 & headache, back pain & surgery (wrapped) & 5 (good) \\
\hline \multirow[t]{2}{*}{ Berlis et al. $(2005)^{1)}$} & $48 / \mathrm{M}$ & $\mathrm{T} 12$ & $\begin{array}{l}\text { abdominal pain, } \\
\text { back pain, anesthesia }\end{array}$ & wait and see & 5 (good) \\
\hline & $69 / \mathrm{F}$ & $\mathrm{T} 12$ & $\begin{array}{l}\text { back pain, } \\
\text { walking impairment }\end{array}$ & wait and see & 5 (good) \\
\hline Massand et al. $(2005)^{15)}$ & $30 / \mathrm{M}$ & T11 & back pain, paresthesias & surgery (wrapped) & 5 (good) \\
\hline Present case & $60 / \mathrm{F}$ & T12 & back pain, lower limb pain & wait and see & 5 (good) \\
\hline
\end{tabular}

F: female, M: male.

reported in $6 \%$ of spinal AVMs and in $75 \%$ to $90 \%$ of cases of AVM associated with aneurysm. ${ }^{3,8)}$ Isolated spinal artery aneurysms not associated with AVM, AVF, or tumor are exceptionally rare. ${ }^{9-11,13,17,19)}$ A review described 57 cases of spinal artery aneurysm. ${ }^{18)}$ Inflammatory processes, arterial dissections, and intimal defects have all been proposed as potential etiologies for these rare isolated lesions. Only 6 cases of ruptured isolated spinal artery aneurysms of the radiculomedullary branch of the artery of Adamkiewicz, including the present case, have been diagnosed by angiography with clinical information available (Table 1). ${ }^{1,5,15,20)}$ The mean age of the patients was 45.2 years (range 30-69 years), with no sex preponderance. Symptoms caused by ruptured spinal artery aneurysms seem to correlate well with the location of the lesion. Sudden neck or back pain, abdominal pain, and motor weakness or sensory disturbance of the lower extremities are suggestive of aneurysms at the thoracolumbar levels.

The pathogenesis of spinal artery aneurysms has been discussed. ${ }^{10,18)}$ Spinal artery aneurysms without inflammatory reaction, infection, or connective tissue disease have also been reported, as in the present case. Most spinal artery aneurysms are small with diameters of $<3 \mathrm{~mm}$, and although both saccular and fusiform aneurysms have been identified, the latter are more frequent. ${ }^{1,3,5,10,12,14,16,18,20,21)}$ Three cases of spinal artery aneurysms were found to be fusiform dissecting lesions at surgery. ${ }^{15)}$ Some spinal artery aneurysms appear to be dissecting lesions. In the present case, spontaneous disappearance of the lesion suggests a dissecting aneurysm. The majority of these aneurysms involved either the artery of Adamkiewicz or the thoracolumbar radicular branches. Our case is unusual because of the location at T12 and the presentation with intracranial SAH.

Our patient presented after rupture of a small fusiform aneurysm supplied by the left radiculomedullary artery at the T12 level. The exact etiology of this aneurysm remains speculative, but we hypothesize that dissection, as primary event, resulted in the formation of a pseudoaneurysm with subsequent hemorrhage. This hemorrhage might have then caused occlusion of the parent artery. However, the aneurysm was collateralized from the left of the T11 segmental artery, as demonstrated during initial diagnostic angiography performed shortly after the SAH occurred. This aneurysm could have been primarily classified as pseudoaneurysm or dissection aneurysm. ${ }^{4)}$
Radicular aneurysms associated with AVM or hyperdynamic flow states have been successfully treated using endovascular and surgical techniques. These types of aneurysms are more amenable to treatment because they are typically associated with robust collateral flow to the anterior spinal artery. ${ }^{13,16)}$ However, no standardized therapy has been established because of the infrequency of SAH from a ruptured isolated spinal artery aneurysm. Therefore, the controversy about clipping and trapping or coil embolization or conservative treatment is not resolved.

Two cases of lateral spinal artery aneurysms were treated with an endovascular technique. ${ }^{3)}$ The endovascular procedure was successful in one case, but surgical intervention was needed in the other. In the present case, we considered that a dissecting aneurysm arising from the artery of Adamkiewicz was not indicated for endovascular trapping because of the absence of distal blood flow. Endovascular treatment may be an appropriate choice, especially if surgical intervention is difficult considering the location of the lesion or the condition of the patient.

Surgical treatment of spinal artery aneurysms may be required, depending on whether or not blood flow is present distal to the lesion, and offers the potential advantage of preservation of the nearby perforators, as well as the possibility of vascular bypass. The anterior spinal artery and the artery of Adamkiewicz must be preserved, and the aneurysm can be resected by direct microvascular reconstruction or may be reinforced with muslin wrapping. ${ }^{3,7,15,20)}$ During the operation, somatosensory and motor evoked potentials must be recorded by tibial nerve stimulation of both lower extremities. Our patient had classical fusiform aneurysm, with no surgical neck and with blood flow distal to the lesion.

In contrast, conservative therapy may be preferred because of the small size of the artery and the presence of distal flow. ${ }^{14)}$ The mass effect of aneurysms or surrounding blood clots may be the only indication for surgical treatment. A 'wait-and-see' strategy was proposed for these aneurysms. ${ }^{1)}$ The differential diagnosis should be directed toward the exclusion of different types of vasculitis and noninflammatory vasculopathies before the initiation of surgical or endovascular treatment. If surgical intervention is delayed for any reason or if emergency surgery is not needed, repeat angiography should be seriously considered before any delayed surgical intervention is 
done because spontaneous regression is likely.

Our case was not amenable to endovascular treatment due to the inaccessibility of the anterior spinal artery. However, surgical clipping or trapping procedure of these aneurysms carries high risk of occluding the anterior spinal artery during the intervention. Limited to isolated spinal dissection aneurysm, treatment guidelines for these aneurysm are not well established, but spontaneous healing of the dissection and obliteration of the aneurysm may be the most likely approach.

The present case of ruptured dissection aneurysm of radiculomedullary branch of the artery of Adamkiewicz with SAH and subsequent spontaneous occlusion indicates that the wait-and-see strategy may be justified and will provide adequate treatment.

\section{References}

1) Berlis A, Scheufler KM, Schmachl C, Rauer S, Gotz F, Schmacher M: Solitary spinal artery aneurysms as a source of spinal subarachnoid hemorrhage: potential etiology and treatment strategy. AJNR Am J Neuroradiol 26: 405-410, 2005

2) Berlis A, Schumacher M, Spreer J, Neumann HP, van Velthoven V: Subarachnoid haemorrhage due to cervical spinal cord haemangioblastomas in a patient with von HippelLindau disease. Acta Neurochir (Wien) 145: 1009-1013, 2003

3) Chen CC, Bellon RJ, Ogilvy CS, Putman CM: Aneurysms of the lateral spinal artery: report of two cases. Neurosurgery 48: 949-954, 2001

4) Djindjian M, Djindjian R, Rey A, Hurth M, Houdart R: Intradural extramedullary spinal arterio-venous malformations fed by the anterior spinal artery. Surg Neurol 8: 85-93, 1977

5) Garcia CA, Dulcey S, Dulcey J: Ruptured aneurysms of the spinal artery of Adamkiewicz during pregnancy. Neurology 29: 394-398, 1979

6) Glasker S, Van Velthoven V: Risk of hemorrhage in hemangioblastomas of the central nervous system. Neurosurgery 57: 71-76, 2005

7) Gonzalez LF, Zabramski JM, Tabrizi P, Wallace RC, Massand MG, Spetzler RF: Spontaneous spinal subarachnoid hemorrhage secondary to spinal aneurysms: diagnosis and treatment paradigm. Neurosurgery 57: 1127-1131, 2005

8) Herdt JR, Di Chiro G, Doppman JL: Combined arterial and arteriovenous aneurysms of the spinal cord. Radiology 99: 589-593, 1971

9) Hino H, Maruyama H, Inomata H: [A case of spinal artery aneurysm presenting transverse myelopathy associated with coarctation of the aorta]. Rinsho Shinkeigaku 29: 1009-1012, 1989 (Japanese)

10) Karakama J, Nakagawa $K$, Maehara $T$, Ohno $K$ : Subarachnoid hemorrhage caused by a ruptured anterior spinal artery aneurysm. Neurol Med Chir (Tokyo) 50: 1015-1019, 2010

11) Kawamura S, Yoshida T, Nonoyama Y, Yamada M, Suzuki A, Yasui N: Ruptured anterior spinal artery aneurysm: a case report. Surg Neurol 51: 608-612, 1999

12) Klinger JH, Glasker S, Shah MJ, Van Velthoven V: Rupture of a spinal artery aneurysm attributable to exacerbated Sjogren syndrome: case report. Neurosurgery 64: E1010-1011, 2009

13) Lavoie P, Raymond J, Roy D, Guilbert F, Weill A: Selective treatment of an anterior spinal artery aneurysm with endosaccular coil therapy. Case report. J Neurosurg Spine 6: 460-464, 2007

14) Longatti P, Sgubin D, Di Paola F: Bleeding spinal artery aneurysms. J Neurosurg Spine 8: 574-578, 2008

15) Massand MG, Wallace RC, Gonzalez LF, Zabramski JM, Spetzler RF: Subarachnoid hemorrhage due to isolated spinal artery aneurysm in four patients. AJNR Am J Neuroradiol 26: 2415-2419, 2005

16) Moore DW, Hunt WE, Zimmerman JE: Ruptured anterior spinal artery aneurysm: repair via a posterior approach. Neurosurgery 10: 626-630, 1982

17) Ohmori Y, Hamada J, Morioka M, Yoshida A: Spinal aneurysm arising from the feeding pedicle of a thoracic perimedullary arteriovenous fistula: case report. Surg Neurol 64: 468-470, 2005

18) Rengachary SS, Duke DA, Tsai FY, Kragel PJ: Spinal arterial aneurysm: case report. Neurosurgery 33: 125-130, 1993

19) Takasaki K, Asakura T, Kadota K, Nakayama M, Komasaku R, Kasamo S: [Spinal artery aneurysm associated with dural AVM of the posterior fossa]. No Shinkei Geka 19: 459-463, 1991 (Japanese)

20) Vishteh AG, Brown AP, Spetzler RF: Aneurysm of the intradural artery of Adamkiewicz treated with muslin wrapping: technical case report. Neurosurgery 40: 207-209, 1997

21) Yahiro T, Hirakawa K, Iwaasa $M$, Tsugu $H$, Fukushima $T$, Utsunomiya $\mathrm{H}$ : Pseudoaneurysm of the thoracic radiculomedullary artery with subarachnoid hemorrhage. Case report. J Neurosurg 100(3 Suppl Spine): 312-315, 2004

Address reprint requests to: Satoshi Iihoshi, MD, Department of Neurosurgery, Sapporo Medical University School of Medicine, South-1st, West-16th, Chuo-ku, Sapporo, Hokkaido 060-8543, Japan. e-mail: iiboshi@sapmed.ac.jp 